\title{
Initial outcomes of mitomycin intravascular chemoembolization (MICE) for corneal neovascularization
}

\author{
Michael Mimouni ${ }^{\mathbb{D}} \cdot$ Dean Ouano
}

Received: 4 December 2021 / Accepted: 16 January 2022 / Published online: 31 January 2022

(C) The Author(s), under exclusive licence to Springer Nature B.V. 2022

\begin{abstract}
Purpose To report on the preliminary outcomes of mitomycin C (MMC) intravascular chemoembolization (MICE) for corneal neovascularization (NV).

Methods This is a retrospective case series of three consecutive eyes that underwent MICE for progressive corneal NV with sight threatening lipid keratopathy. A $1.0 \mathrm{cc}$ syringe was partially filled with MMC $(0.4 \mathrm{mg} / \mathrm{mL})$ and attached to a 33-gauge needle used to cannulate the vessels. The MMC (0.01-0.05 ml) was injected with enough retrograde hydrostatic force to fill efferent and afferent vessels. Follow-up ranged from 4 months to 1 year.
\end{abstract}

Results Three eyes of three patients aged 59, 73 and 33 years were included. There were no intraoperative or postoperative complications associated

Supplementary Information The online version contains supplementary material available at https://doi. org/10.1007/s 10792-022-02240-6.

M. Mimouni $(\bowtie)$

Department of Ophthalmology and Vision Sciences, University of Toronto, Toronto, Canada

e-mail:michael@intername.co.il

M. Mimouni

Department of Ophthalmology, Rambam Health Care

Campus, Affiliated with the Bruce and Ruth Rappaport

Faculty of Medicine, Technion-Israel Institute of

Technology, Haifa, Israel

D. Ouano

Coastal Eye Clinic, New Bern, NC, USA with the MICE procedure. Patient 1 presented with progressive corneal $\mathrm{NV}$ and lipid keratopathy secondary to herpes zoster ophthalmicus (HZO) and a best-corrected spectacle visual acuity (BSCVA) of 20/100 Snellen. At one-year post-MICE, there was no recurrence (BSCVA was 20/20 Snellen). Patient 2 presented with idiopathic lipid keratopathy (BSCVA 20/50 Snellen). At four months post-MICE, there were no signs of recurrence (BSCVA 20/20 Snellen). Patient 3 presented with corneal NV and lipid keratopathy secondary to HZO (BSCVA 20/30 Snellen). At four months following two MICE treatments, resolution of the lipid keratopathy was noted (BSCVA 20/20 Snellen).

Conclusions Preliminary findings suggest that MICE may be an additional modality for treating progressive corneal NV with lipid keratopathy. Larger comparative studies with longer follow-up are warranted.

Keywords Mitomycin - Intravascular · Chemoembolization - Corneal $\cdot$ Neovascularization . Lipid keratopathy

\section{Introduction}

The absence of blood vessels in the cornea is a necessity for optical clarity and optimal vision. Corneal neovascularization (NV) is a vision-threatening pathologic response to a diverse number of infectious, 
traumatic, ischemic and foreign body associated etiologies [1]. Permanent visual loss is related to lipid deposition, scarring, irregular astigmatism and loss of corneal clarity $[2,3]$. The incidence of corneal NV varies based on geographic location and population and was previously estimated at 1.4 million patients per year in 1998, [4], likely increasing since then [1].

The primary approach to corneal NV is the treatment of the underlying infectious and inflammatory stimulus, depending on the disease process [3]. Topical steroids are a commonly used treatment, but adverse effects associated with prolonged use limit its long-term use. There is little evidence that topical steroids reverse established corneal vascularization $[5,6]$. A wide variety of vasodestructive treatments including argon laser photocoagulation, photodynamic therapy, cautery, fine needle diathermy and suture ligation have met with relatively poor longterm success. More recently use of topical and subconjunctival anti-vascular endothelial growth factor (anti-VEGF) has been described [1]. A meta-analysis that included twenty-five clinical and experimental studies reported a significant reduction of corneal NV after treatment with either topical or subconjunctival bevacizumab [7]. The rate of recurrence of corneal NV and lipid keratopathy after subconjunctival bevacizumab has been reported to be $35 \%$ in one published series [8].

The efficacy of intravascular chemoembolization with various agents has been reported for hepatocellular carcinoma [9] with mitomycin C (MMC) being one of the first [10]. It is likely that MMC inhibits vascular endothelial cell proliferation, thereby limiting repair capacity of the endothelial monolayers of the blood vessel $[11,12]$. This led to the novel hypothesis that MMC intravascular chemoembolization (MICE) may serve as a potentially effective treatment for corneal NV. The purpose of the current study was to report on the short-term outcomes of MMC intravascular chemoembolization for corneal NV (MICE) in three patients.

\section{Methods}

This study was conducted in compliance with the tenets of the Declaration of HELSINKI. All patients signed informed consent prior to undergoing the procedure, and further consent was obtained for inclusion in this report. Patients were advised that most experience with transarterial chemoembolization has come from treating hepatocellular carcinoma and although a minute amount $(0.02 \mathrm{mg})$ was going to be injected, this may lead to side effects and to contact the surgeon and their general practitioner immediately if needed. Additionally, they were advised that this may not succeed and that they may require repeat injections and that other agents may need to be administered in future (e.g., bevacizumab).

Study participants

The MICE treatment was performed on three patients from the same facility (Coastal Eye Clinic) by the same surgeon (DPO). The study's specific inclusion criteria were the prevention of visual loss in patients with progressive lipid keratopathy due to corneal neovascularization. This is a retrospective case series conducted utilizing a chart review of consecutive eyes that underwent MICE for corneal NV from November 2019 to September 2020. Inclusion criteria for MICE treatment were (a) Lipid keratopathy secondary to corneal neovascularization encroaching the visual axis, (b) Documented failure of conventional treatment of the etiology of corneal NV, specifically systemic anti-viral therapy (valacyclovir) for a minimum of 4 months, (c) A previous trial of topical steroids for at least one month with persistent NV, (d) No prior corneal vasodestructive or anti-proliferative therapy, (e) Follow-up examinations ranged from 3 months to one year.

\section{Pre-treatment assessment}

All patients underwent the following assessment before MICE treatment: ophthalmic examination, slit lamp imaging $(\times 16$ and $\times 25$ magnification), corneal topography and anterior segment optical coherence tomography (AS-OCT) imaging.

\section{Surgical technique}

All patients underwent MICE using a similar protocol with the aid of an ophthalmic operating room microscope. Topical anesthesia in the form of proparacaine or preservative-free lidocaine was used, and 5\% povidone iodine solution was then instilled on the ocular surface. A $1.0 \mathrm{cc}$ syringe was partially filled with 
MMC (0.4 mg/mL) and attached to a 33-gauge needle (TSK Laboratory International, Canada, Vancouver). The largest bore corneal vessel just inside the limbus was identified (Fig. 1 white arrow). The needle was angled at approximately 15 degrees from the corneal surface to cannulate the vessel. A small volume of MMC (0.01 to $0.05 \mathrm{ml})$ was injected with enough retrograde hydrostatic force to fill both the efferent and afferent vessels (Fig. 1 black arrow). No more than a total volume of $0.05 \mathrm{ml}$ of mitomycin was injected intravascularly in any case. Balanced salt solution was used for meticulous irrigation of the ocular surface (to remove any remnant ocular surface MMC) and one drop of moxifloxacin $0.5 \%$ and prednisolone acetate $1 \%$ was applied at the end of the procedure.

\section{Post-treatment assessment}

All patients were followed up at 1 day, 1 month, 3 months and 6 months following the procedure. At every visit, they underwent the following assessment: ophthalmic examination, slit lamp imaging $(\times 16$ and $\times 25$ magnification), tomography and anterior segment optical coherence tomography imaging.

\section{Documentation of corneal NV}

Photographs taken at each visit were graded by 2 masked observers for extent (1 to 12 clock hours), centricity (peripheral, paracentral and involving visual axis) and density (very low, low, moderate and high) of corneal NV as previously described [13].
The amount of vascularization was measured on the photographs as the percentage of the total area of the cornea by using an image processing and analysis software program (Image J v1.8.0_112, Javabased image processing, NIH, Bethesda, Maryland, USA).

\section{Statistical analysis}

Data were analyzed with the Minitab Software, version 17 (Minitab Inc, State College, PA). For the analysis of paired continuous data, the Wilcoxon Signed rank test was used. In all analyses, a twosided $p$ value $<0.05$ was considered statistically significant. All presented means are accompanied by their respective standard deviations.

\section{Results}

A total of three eyes of three patients aged 33, 59 and 73 years all of which were females were included in this study. No complications during or postMICE treatment were noted. The mean pre-treatment vascularization area was $9.77 \pm 2.45 \%$ (range $7.68 \%-12.46 \%$ ). Following treatment, the area of active vascularization was reduced to $0.39 \pm 0.67 \%$ (range $0-1.16 \%)(p=0.03)$. There was no evidence of loss of normal limbal vascularization in any of the cases throughout the follow-up period.
Fig. 1 The largest bore corneal vessel just inside the limbus was identified (white arrow). The needle was angled at approximately 15 degrees from the corneal surface to cannulate the vessel. A small volume of MMC ( 0.01 to $0.05 \mathrm{ml})$ was injected with enough retrograde hydrostatic force to fill both the efferent and afferent vessels (black arrow)

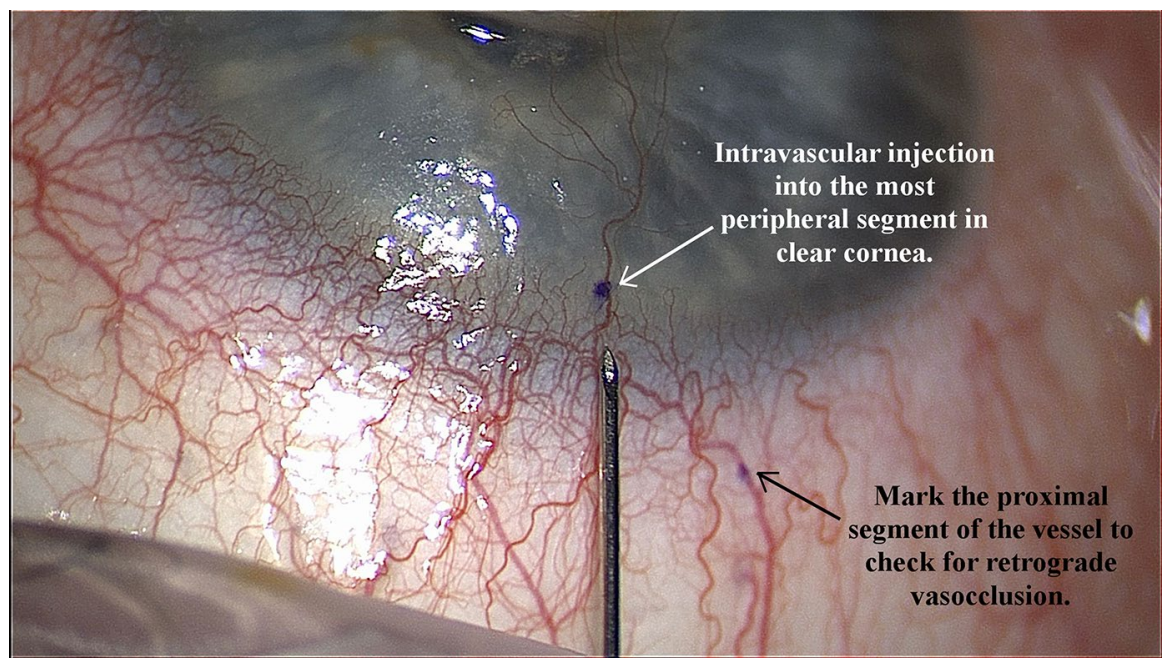




\section{Patient \#1}

An otherwise healthy 59-year woman developed herpes zoster ophthalmicus (HZO) in her right eye four years before presentation despite receiving a Zostavax (Merck, Readington Township, New Jersey, USA) vaccination seven years prior to presentation. She was originally treated with valacyclovir but still progressed to early pseudodendritic and stromal keratitis. For 3 years, she was stabilized with chronic valacyclovir $500 \mathrm{mg}$ twice daily and variable dosing of topical prednisolone acetate and fluorometholone but still developed progressive corneal NV and lipid keratopathy (Fig. 2A and B). Her best-corrected spectacle visual acuity (BSCVA) was 20/100 Snellen at presentation. She underwent uneventful MICE treatment protocol. At postoperative visit day 1, her BSCVA improved to $20 / 30$ and complete obliteration of the corneal NV was documented (Fig. 2C). No recurrence of the corneal NV occurred at the 3 weeks (Fig. 2D) and at the 8-week follow-up absorption of the lipid was noted (Fig. 2E), and BSCVA was reduced to 20/80 Snellen secondary to thinning and flattening in the area of the absorption of lipid (Fig. 3 and Supplement 1). At one-year post-treatment, there was no recurrence of corneal $\mathrm{NV}$ and BSCVA improved to 20/20 Snellen (Fig. 2F).

\section{Patient \#2}

An otherwise healthy 73-year-old female who presented with idiopathic lipid keratopathy (Fig. 4A and $\mathrm{B}$ ), which rapidly progressed over a period of two months. There was no known history of HZO or herpes simplex, prior contact lens wear, trauma or Rosacea. Her BSCVA at presentation was 20/50.
Fig. 2 Patient \# 1. At presentation (A, B) her BSCVA was 20/100. At postoperative day $1(\mathbf{C})$ complete obliteration of corneal NV noted. No recurrence noted at 3 weeks (D) and absorption of lipid noted at 8 weeks (E). At one-year no recurrence of corneal NV (F)
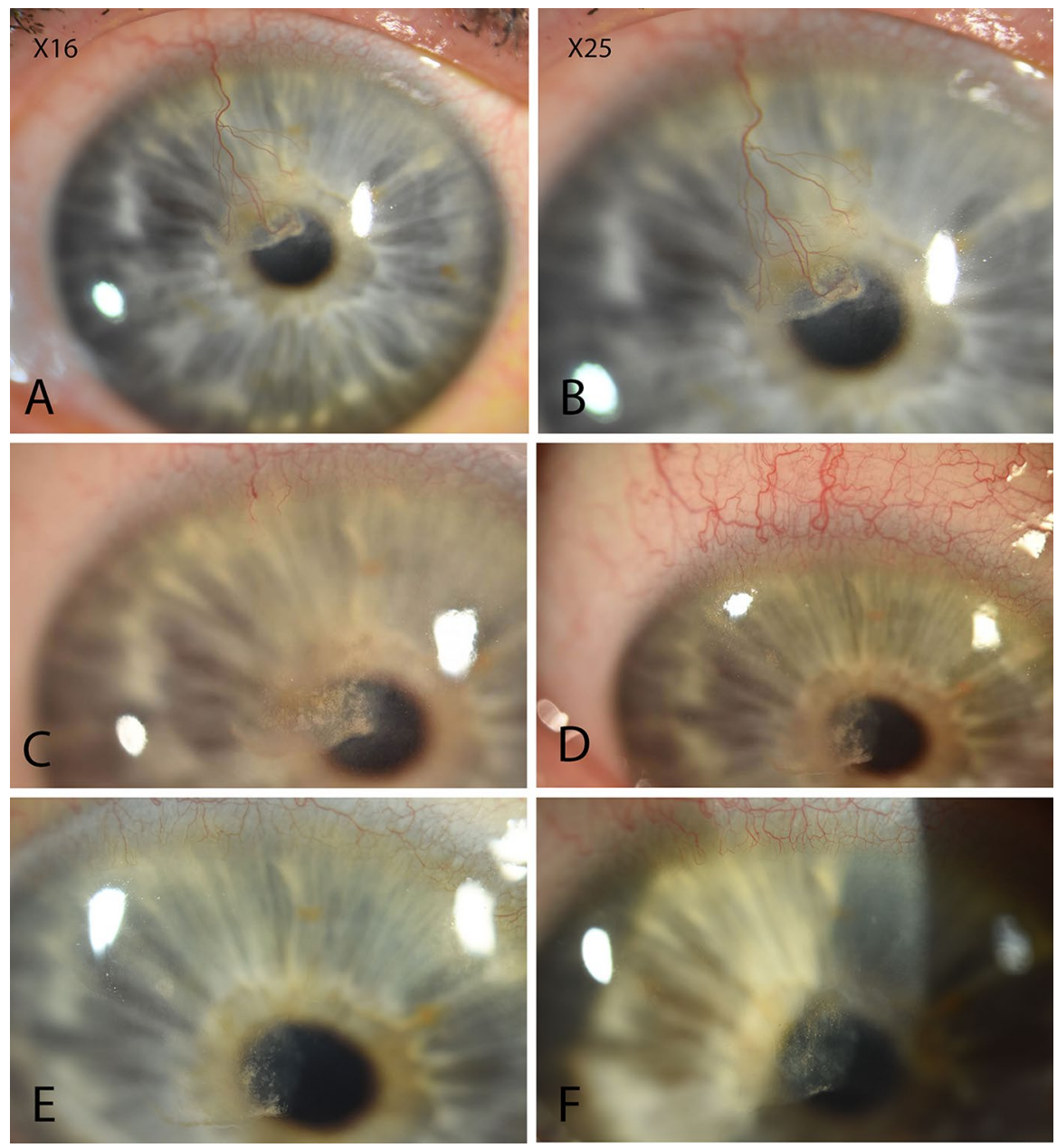
Fig. 3 Anterior segment optical coherence tomography of all three patients before and after treatment. Thinning of the involved areas may be noted with more compact and overall reduction of hyper-reflectivity following treatment when compared to before treatment

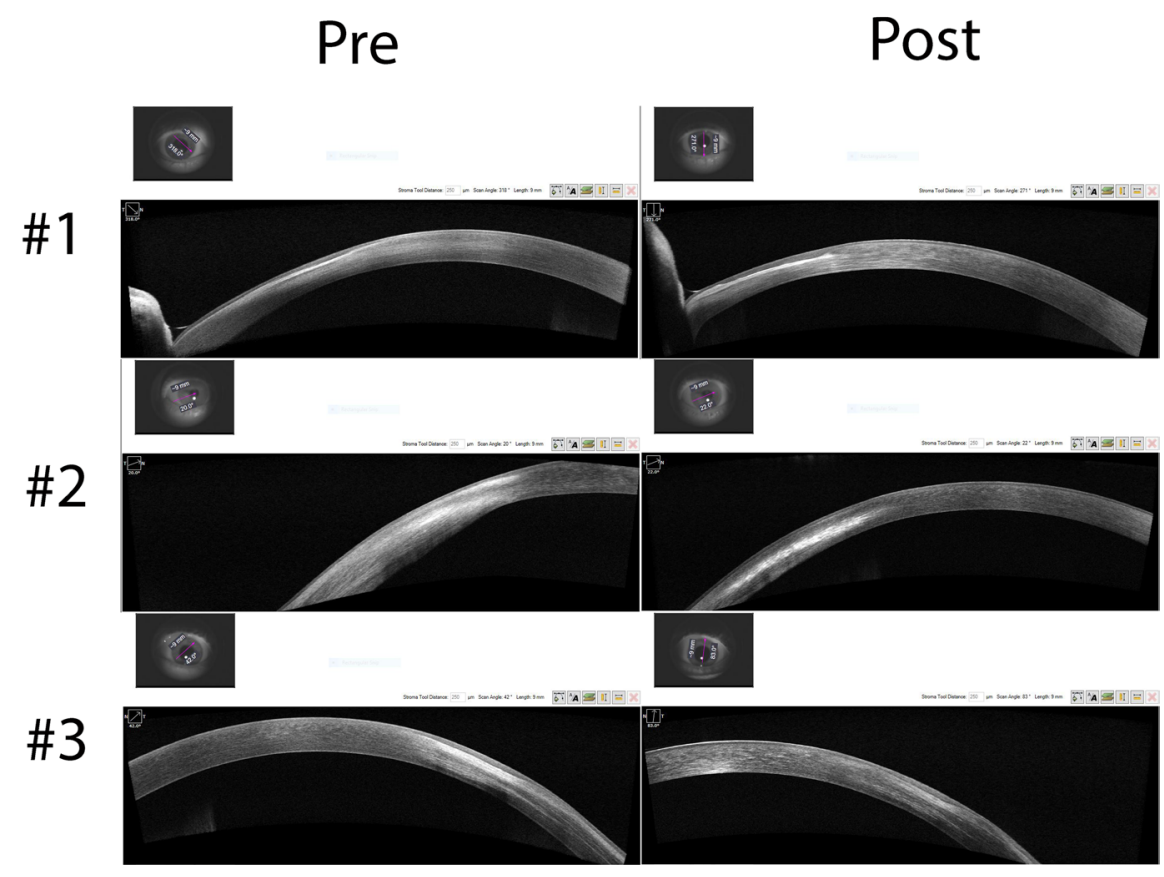

She was initially treated empirically on valacyclovir $1 \mathrm{~g}$ three times a day as well as topical steroids with no improvement. Serum lipid profile was tested and found to be normal. Surgical intervention was delayed by a positive screening nasopharyngeal swab for COVID-19. A week later she was retested and was COVID-19 negative with positive serum antibodies. She subsequently underwent uneventful MICE treatment protocol. At the 10-day postoperative visit, her BSCVA was 20/30, and the peripheral cornea revealed areas of lipid and blood trapped in the corneal stroma which we coined "pizza pie" sign (Fig. 4C and D). At four months post-treatment BSCVA improved to 20/20 Snellen, and there were no signs of corneal NV. The density of the lipid continued to absorb leading to a feathery like remnant of opacities (Fig. 4E). The reduction in density of the lipid from baseline (Fig. 4F) is identified on AS-OCT as well (Fig. 3).

\section{Patient \#3}

An otherwise healthy 33-year-old female who developed HZO with lipid keratopathy in her left eye two years prior to presentation (Fig. 5A and B). She noted progressive loss of vision over that period of time despite oral valacyclovir and topical steroids.
Her BSCVA at presentation was 20/30 Snellen. She underwent a first uneventful MICE treatment protocol which led to partial resolution of the blood vessels and lipid deposits. However, remnant high flow blood vessels were identified at the two month postoperative visit (Fig. 5C and D). She underwent a second MICE treatment using the same protocol. At four months following, the second MICE treatment resolution of the lipid keratopathy was noted, and her BSCVA improved to 20/20 (Fig. 5E and F).

\section{Discussion}

The current study describes the outcomes of three patients with progressive vision loss due to lipid keratopathy secondary to persistent corneal NV that were treated with MICE protocol. In all three cases, the MICE treatment led to resolution of the corneal $\mathrm{NV}$ and subsequent partial absorption of the lipid keratopathy. All three patients achieved a BSCVA of 20/20 Snellen, and no intraoperative or postoperative complications post-MICE were noted. To the best of our knowledge, this is the first report of intravascular chemoembolization for the treatment of corneal NV.

In the current study, two patients required one MICE treatment to ablate all of the high velocity 
Fig. 4 Patient \#2. Preoperative color photographs (A, B). Ten days post-treatment demonstrating the "pizza pie" sign $(\mathbf{C}, \mathbf{D})$ representing trapped lipid and blood. Four months post-treatment demonstrating absorption of lipid and feathery like remnants $(\mathbf{E})$
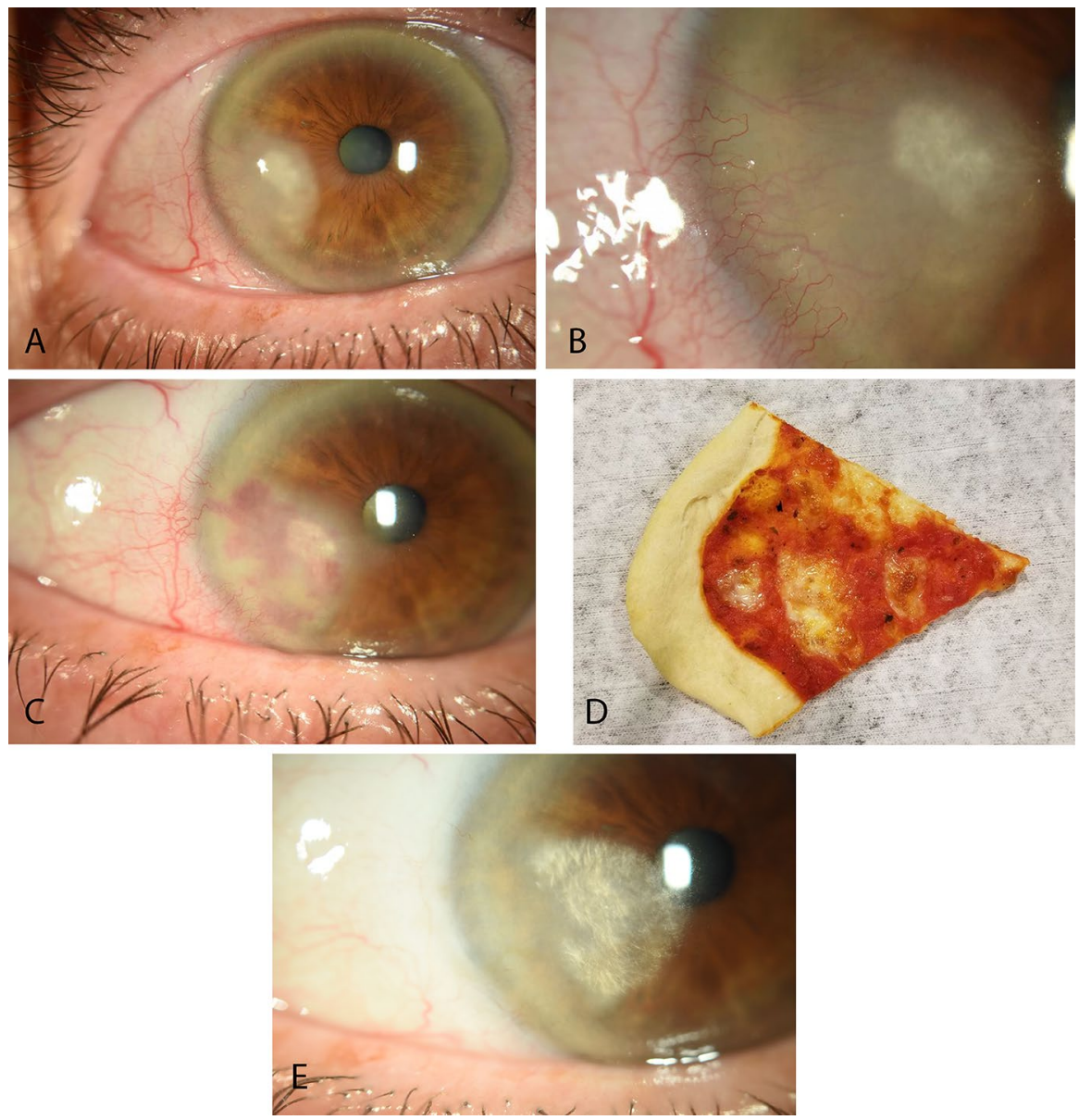

blood vessels. One patient (\#3) demonstrated residual high velocity blood vessels, and therefore, a second MICE treatment was performed, and following the second MICE treatment resolution of high velocity blood vessels was safely achieved as well. We speculate that in patient \#3, deeper high velocity blood vessels were not successfully chemoembolized, and therefore were still seen at two months following the first treatment. Therefore, MICE was repeated a second time with no adverse outcomes indicating that MICE may be safely repeated when necessary. As this procedure is technically challenging, a visual cue that surgeons can look for during the procedure to verify that the MMC was indeed injected intravascular is to look for blanching of the blood vessels during the injection. If a vessel does not blanch during the procedure, it is likely that the MMC has gone intrastromal and not into the blood vessel itself. Therefore, to successfully cannulate the vessel particular attention should be given toward proper angling of the needle (approximately 15 degrees). Furthermore, the entire bevel of the needle must be inserted into stroma otherwise the MMC will go to the path of least resistance (ocular surface) and not into the vessel. On the other hand, the surgeon must avoid complete penetration of full thickness cornea as injection of MMC into the anterior chamber could have disastrous consequences [14]. Finally, only a small volume of MMC $(0.01-0.05 \mathrm{ml})$ is needed to fill both the efferent and afferent vessels during the injection when located in the correct position and angle.

In all three cases, following successful embolization of the corneal NV, a common sequence of events was noted. Early postoperative findings include ablation of the corneal $\mathrm{NV}$ which can be witnessed the following day. Afterward at 1-3 weeks following treatment, the "pizza pie" sign can be identified, indicating areas in which lipid and blood are trapped in 
Fig. 5 Patient \#3. Preoperative color photographs (A, B). Two months posttreatment remnant high velocity blood vessels are still present $(\mathbf{C}, \mathbf{D})$, and therefore, treatment was repeated. Four months postsecond MICE treatment absorption of lipid and no recurrence of corneal $\mathrm{NV}$ $(\mathbf{E}, \mathbf{F})$
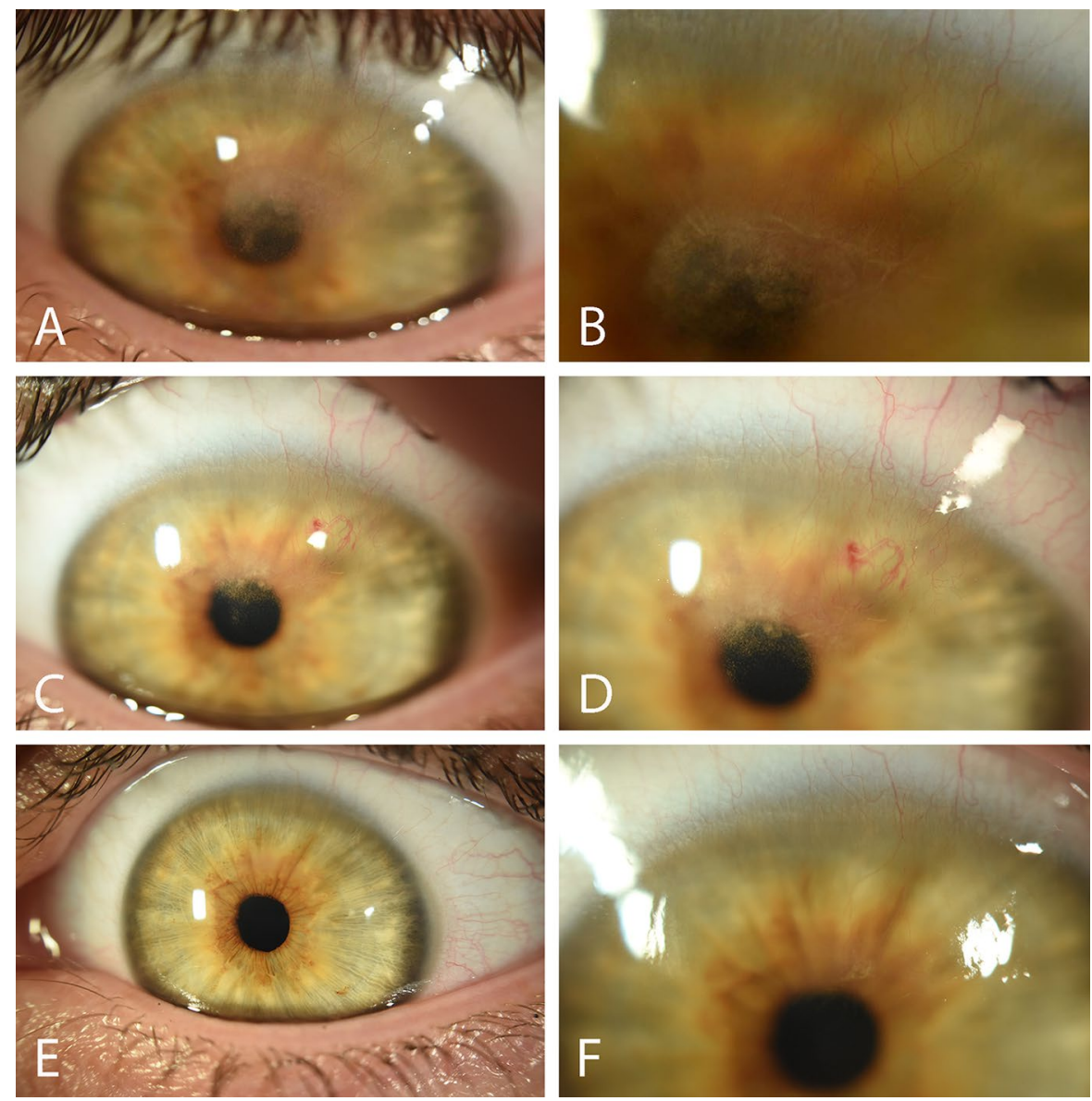

the corneal stroma. After 1-2 months, absorption of lipid ensues leading to a feathery like appearance of the left-over lipid with reduced hyper-reflectivity on AS-OCT. This absorption may be accompanied by compaction or flattening of the cornea noted in the area of the absorbed lipid with induced astigmatism. However, the corneal flattening and induced astigmatism stabilized or improved in all cases. There was no loss of best-corrected vision or recurrence of corneal neovascularization in any case during the study period.

Regarding potential safety concerns. A recent study assessing two different types of transarterial chemoembolization with mitomycin for hepatocellular carcinoma reported on outcomes for concentrations of $8 \mathrm{mg} / \mathrm{m}^{2}$ body surface [15]. The average body surface of an adult is $1.6-1.9 \mathrm{~m}^{2}$ indicating a total of $12.8-15.2 \mathrm{mg}$ in their cohort of 99 patients [15]. For comparison, with MICE, 0.01-0.05 $\mathrm{ml}$ of $0.4 \mathrm{mg} / \mathrm{ml}$ mitomycin is injected, which means that at most $0.02 \mathrm{mg}$ is injected. Effectively, at most, $\sim 0.16 \%$ $((0.02 \mathrm{mg} / 12.8 \mathrm{mg}) * 100)$ of what is injected for hepatocellular carcinoma is injected during MICE. In the aforementioned study on hepatocellular carcinoma, the majority of patients tolerated chemoembolization well and all patients were discharged from the hospital on the day of treatment [15]. At that high dosage, given to the hepatic artery, approximately $15 \%$ develop transient symptoms of abdominal pain, nausea, and vomiting for several days ("postembolization syndrome"). It is speculated that this is due to an inflammatory response due to breakdown of the tumor tissue. No major complications or allergic reactions were reported. At the microscopic dosages given for MICE $(0.16 \%)$ for corneal neovascularization, we have not witnessed any systemic adverse events. In the current study, there was no evidence of loss of normal limbal vascularization in any of the cases throughout the follow-up period. We strongly recommend avoiding injecting $\mathrm{MMC}$ toward the 
limbus when performing MICE in order to avoid this potential unwanted effect. We also recommend meticulously irrigating the ocular surface with BSS at the end of the procedure to reduce ocular surface exposure to MMC.

It is thought that newly formed blood vessels bud from pre-existing blood vessels or vascular endothelial progenitor cells. Moreover, lymphatic vessels bud from pre-existing lymphatic vessels or blood vessels [16]. In its non-pathological state, the cornea maintains a state of "corneal angiogenic privilege" (avascular status) [17]. A well preserved equilibrium between anti-angiogenic and pro-angiogenic mechanisms prevents neovascularization onto the resting cornea [17]. Following injury in the form of infection, trauma or inflammation, this equilibrium is disturbed leading to a pro-angiogenic state [17]. Some of these pro-angiogenic factors such as VEGF stimulate vascular endothelial cell proliferation [18]. Subsequently, these endothelial cells migrate toward these angiogenic stimuli forming new lumens, branches and eventual loops with afferent and efferent limbs [19]. Indeed, topical and subconjunctival anti-VEGF treatment such as bevacizumab [7], ranibizumab [20] and aflibercept [21, 22] have been extensively reported. However, corneal NV may recur [8] or persist [23] following treatment leading to repeated injections. It has been proposed that this is due to residual subclinical inflammation and rebound release of VEGF after cessation of treatment [8]. Furthermore, other growth factors and cytokines have been proposed as potential target of therapies [24]. An additional treatment modality, fine needle diathermy, has been reported as a useful and inexpensive technique for the treatment of established corneal vessels. In a series of 14 eyes, Pillai et al. reported occlusion of all vessels in 57.1\% $(n=8)$ with the remainder achieving at least $50 \%$ occlusion [25]. More recently, Schaub et al. reported on regression of pathological corneal neovascularization prior to high risk keratoplasty using corneal crosslinking. In a series of five patients, they reported a mean reduction of $70.5 \%$ in mean corneal neovascularization with no incidences of revascularization observed [26]. The use of chemotherapeutic agents to has been shown to provide adequate vessel occlusion in other fields such as the treatment of hepatocellular carcinoma [9]. With MICE, the vessels endothelial cells are obliterated, and existing abnormal corneal vessels are occluded and subsequently ablated. We speculate that targeting of the vessel and its endothelium by means of chemotherapeutic embolization not only halts and destroys existing vessels but also prevents further vascularization [24]. Mitomycin is a bifunctional alkylating agent that is known to have direct cytotoxic effects on cultured pulmonary artery endothelial cells and umbilical vein endothelial cells $[11,12]$. Unlike topical steroid or anti-VEGF agents, the endothelial cell cycle arrest from mitomycin is irreversible. Other potential corneal NV chemoembolization agents exist, however, the ideal one is currently unknown and future studies may compare results with MICE.

Performing MICE can be technically challenging, especially in cases of smaller vessels. Therefore, we recommend identifying the largest vessel (regardless of whether it's an afferent or efferent vessel). The hydrostatic pressure from the injection itself with fill the entire network with MMC including the smallest vessels, if they are connected. Indeed, there are some instances where even the largest vessel is smaller than the diameter of a 34-gauge needle itself. In those cases, placing the needle tip sufficiently intrastromal, where the tip is fully buried, at the entrance of a vessel will cause the MMC to go to the path of least resistance when injecting and therefore into the vessel itself rather than into the stroma or onto the ocular surface. Finally, indeed in some cases, this may require several attempts with some of the MMC going intrastromal and not into the vessel itself.

This preliminary case series has several limitations. First of which is its retrospective nature. Second, AS-OCT angiography or fluorescein angiography could have added valuable information as to the effect of the treatment and were not performed. Therefore, future studies assessing MICE may consider adding this modality. Third, specular microscopy was not performed in this study since the lipid was threatening their visual axes, and the media was too opaque. Future studies may consider attempting central endothelial cell counts and, if not possible, peripheral cell counts in areas of clear cornea.

In summary, this study presents the outcomes of MICE for lipid keratopathy secondary to corneal NV. All three cases achieved complete resolution of corneal NV and subsequent partial absorption of the lipid keratopathy with no intraoperative or postoperative complications noted. Therefore, preliminary findings suggest that MICE may be an additional 
modality for treating this entity for appropriate cases. This treatment is technically challenging to perform, and larger comparative studies with longer follow-up are warranted to assess its safety and efficacy.

Author Contributions All authors contributed to the study conception and design. Material preparation, data collection and analysis were performed by Michael Mimouni and Dean Ouano. The first draft of the manuscript was written by Michael Mimouni and Dean Ouano. All authors read and approved the final manuscript.

Funding The authors declare that no funds, grants or other support were received during the preparation of this manuscript.

\section{Declarations}

Conflict of interest The authors have no relevant financial or non-financial interests to disclose.

Ethical approval All procedures performed in studies involving human participants were in accordance with the ethical standards of the institutional and/or national research committee and with the 1964 Declaration of HELSINKI and its later amendments or comparable ethical standards. Given the retrospective nature of this limited case series $(n=3)$, an IRB approval was not required.

Consent to Participate Written formal consent was obtained from all subjects.

Consent to Publish Written formal consent was obtained from all subjects.

\section{References}

1. Abdelfattah NS, Amgad M, Zayed AA, Salem H, Elkhanany AE, Hussein H, Abd El-Baky N (2015) Clinical correlates of common corneal neovascular diseases: a literature review. Int J Ophthalmol 8:182-193

2. Shakiba Y, Mansouri K, Arshadi D, Rezaei N (2009) Corneal neovascularization: molecular events and therapeutic options. Recent Pat Inflamm Allergy Drug Discov 3:221-231

3. Feizi S, Azari AA, Safapour S (2017) Therapeutic approaches for corneal neovascularization. Eye Vis (Lond) 4:28

4. Dana MR, Schaumberg DA, Kowal VO, Goren MB, Rapuano CJ, Laibson PR, Cohen EJ (1995) Corneal neovascularization after penetrating keratoplasty. Cornea 14:604-609

5. Cursiefen $\mathrm{C}$, Wenkel $\mathrm{H}$, Martus $\mathrm{P}$, Langenbucher A, Nguyen NX, Seitz B, Kuchle M, Naumann GO (2001) Impact of short-term versus long-term topical steroids on corneal neovascularization after non-high-risk keratoplasty. Graefes Arch Clin Exp Ophthalmol 239:514-521
6. Al-Torbak A, Al-Amri A, Wagoner MD (2005) Deep corneal neovascularization after implantation with intrastromal corneal ring segments. Am J Ophthalmol 140:926-927

7. Papathanassiou M, Theodoropoulou S, Analitis A, Tzonou A, Theodossiadis PG (2013) Vascular endothelial growth factor inhibitors for treatment of corneal neovascularization: a meta-analysis. Cornea 32:435-444

8. Chu HS, Chen TC, Hu FR, Chen WL (2013) Recurrence of corneal neovascularization associated with lipid deposition after subconjunctival injection of bevacizumab. Cornea 32:1446-1453

9. Marelli L, Stigliano R, Triantos C, Senzolo M, Cholongitas E, Davies N, Tibballs J, Meyer T, Patch DW, Burroughs AK (2007) Transarterial therapy for hepatocellular carcinoma: which technique is more effective? A systematic review of cohort and randomized studies. Cardiovasc Intervent Radiol 30:6-25

10. Hidaka H, Kobayashi H, Ohyama M, Maeda T, Ikeda K, Nakajo M, Oyama T, Sonoda T, Ito Y, Shinohara S (1985) Transarterial chemoembolization therapy of hepatocellular carcinoma using anticancer agents (mitomycin C and/or adriamycin) suspended in lipiodol. Nippon Igaku Hoshasen Gakkai Zasshi 45:1430-1440

11. Hoorn CM, Wagner JG, Petry TW, Roth RA (1995) Toxicity of mitomycin $\mathrm{C}$ toward cultured pulmonary artery endothelium. Toxicol Appl Pharmacol 130:87-94

12. Seki Y, Toba K, Fuse I, Sato N, Niwano H, Takahashi H, Tanabe N, Aizawa Y (2005) In vitro effect of cyclosporin $\mathrm{A}$, mitomycin $\mathrm{C}$ and prednisolone on cell kinetics in cultured human umbilical vein endothelial cells. Thromb Res 115:219-228

13. Bahar I, Kaiserman I, McAllum P, Rootman D, Slomovic A (2008) Subconjunctival bevacizumab injection for corneal neovascularization. Cornea 27:142-147

14. Wu KY, Hong SJ, Huang HT, Lin CP, Chen CW (1999) Toxic effects of mitomycin-C on cultured corneal keratocytes and endothelial cells. J Ocul Pharmacol Ther 15:401-411

15. Gruber-Rouh T, Schmitt C, Naguib NNN, Nour-Eldin NA, Eichler K, Beeres M, Vogl TJ (2018) Transarterial chemoembolization (TACE) using mitomycin and lipiodol with or without degradable starch microspheres for hepatocellular carcinoma: comparative study. BMC Cancer 18:188

16. Bock F, Maruyama K, Regenfuss B, Hos D, Steven P, Heindl LM, Cursiefen C (2013) Novel anti(lymph)angiogenic treatment strategies for corneal and ocular surface diseases. Prog Retin Eye Res 34:89-124

17. Di Zazzo A, Gaudenzi D, Yin J, Coassin M, Fernandes M, Dana R, Bonini S (2021) Corneal angiogenic privilege and its failure. Exp Eye Res 204:108457

18. Ellenberg D, Azar DT, Hallak JA, Tobaigy F, Han KY, Jain S, Zhou Z, Chang JH (2010) Novel aspects of corneal angiogenic and lymphangiogenic privilege. Prog Retin Eye Res 29:208-248

19. Faraj LA, Said DG, Al-Aqaba M, Otri AM, Dua HS (2016) Clinical evaluation and characterisation of corneal vascularisation. Br J Ophthalmol 100:315-322

20. Turkcu FM, Cinar Y, Turkcu G, Sahin A, Cingu AK, Yuksel H, Sahin M, Yildirim A, Caca I (2014) Topical and subconjunctival ranibizumab (lucentis) for corneal 
neovascularization in experimental rat model. Cutan Ocul Toxicol 33:138-144

21. Sella R, Gal-Or O, Livny E, Dachbash M, Nisgav Y, Weinberger D, Livnat T, Bahar I (2016) Efficacy of topical aflibercept versus topical bevacizumab for the prevention of corneal neovascularization in a rat model. Exp Eye Res 146:224-232

22. Gal-Or O, Livny E, Sella R, Nisgav Y, Weinberger D, Livnat T, Bahar I (2016) Efficacy of subconjunctival aflibercept versus bevacizumab for prevention of corneal neovascularization in a rat model. Cornea 35:991-996

23. Sella R, Ben Ishai M, Livny E, Nahum Y, Bahar I (2021) Subconjunctival aflibercept for the treatment of formed corneal neovascularization. Eye Contact Lens 47:180-184

24. Hall MN, Moshirfar M, Amin-Javaheri A, Ouano DP, Ronquillo Y, Hoopes PC (2020) Lipid keratopathy: a review of pathophysiology, differential diagnosis, and management. Ophthalmol Ther 9:833-852

25. Pillai CT, Dua HS, Hossain P (2000) Fine needle diathermy occlusion of corneal vessels. Invest Ophthalmol Vis Sci 41:2148-2153

26. Schaub F, Hou Y, Zhang W, Bock F, Hos D, Cursiefen C (2021) Corneal crosslinking to regress pathologic corneal neovascularization before high-risk keratoplasty. Cornea 40:147-155

Publisher's Note Springer Nature remains neutral with regard to jurisdictional claims in published maps and institutional affiliations. 